\title{
Consideraciones acerca de la bibliografía del metodismo en México
}

\author{
Rubén Ruiz Guerra
}

\section{Introducción}

41 tiempo de la consolidación de la república restaurada, México presenció un fenómeno cuyas consecuencias podemos ver hasta nuestros días: la introducción de creencias no católicas en materia religiosa. $\mathrm{Al}$ amparo de la ley de libertad de cultos y después de un intento gubernamental por crear una Iglesia netamente mexicana, varias sociedades misioneras norteamericanas iniciaron trabajos en nuestro país.

Para ese momento ya se habían hecho esfuerzos por divulgar este tipo de ideas al sur del rio Bravo. Con el inicio de la vida independiente, los mexicanos conocieron el trabajo de representantes de las sociedades bíblicas inglesa y norteamericana. Durante la guerra del cuarenta y siete, los soldados y los capellanes del ejército invasor repartieron Biblias y porciones de las Escrituras. En la década del cincuenta decimonónico, misioneros norteamericanos independientes cruzaron la frontera y llegaron tan lejos como Zacatecas predicando su particular forma de creencia religiosa.

Estas empresas, debido al escaso apoyo institucional que recibieron y a la misma resistencia de los mexicanos, tuvieron poco éxito. No obstante, se considera que prepararon el terreno para la siembra del protestantismo en "tierra azteca". Es un hecho que las congregaciones o los grupos de estudio que se formaron a raíz de estos esfuerzos respondieron al llamado de los misioneros para establecer nuevas iglesias durante los años setenta. Esos grupos fueron los que hicieron el "llamado macedónico" que aducían los predicadores norteamericanos para justificar su presencia en tierra ya cristiana.

De todas las sociedades misioneras que iniciaron trabajos en México durante el gobierno de Lerdo, probablemente la más importante fue la Metodista Episcopal. ${ }^{1}$ Aunque no fue la primera Iglesia protestante en llegar a nuestro país, sí fue la que se colocó a la cabeza del proceso evangelizador. Diversos voceros metodistas sostienen que la suya fue la denominación que alcanzó mayor número de creyentes y la que dio origen a las organizaciones e instituciones más influyentes dentro del ámbito protestante mexicano. En los últimos años del siglo XIX se considera que el veinticinco por ciento de la propiedad detentada por las sociedades misioneras pertenecía a estos metodistas.

También en 1873 la Iglesia Metodista Episcopal del Sur inició traba-

${ }^{1}$ En 1844 la principal Iglesia metodista de los Estados Unidos, la Metodista Episcopal, se dividió a causa del problema de la esclavitud. Resultado de ello fue la creación de una rama "norteña" del metodismo, la Iglesia Metodista Episcopal, y una rama "sureña", la Iglesia Metodista Episcopal del Sur. Ambas iglesias abrieron misión en México en 1873. 
jos en México y aunque nunca llegó a tener la importancia de su Iglesia hermana, alcanzó altas cifras de crecimiento tanto en propiedades como en número de creyentes a ella adscritos. Si consideramos en conjunto la membresía e influencia de ambos metodismos, nos daremos cuenta que la suya fue la forma más extendida de adoración entre los mexicanos no católicos hasta bien entrado el sigloxX.

La prédica traída por los misioneros norteamericanos era particularmente atractiva para la sociedad mexicana del último cuarto del siglo XIX: A la fe en Dios y a la necesidad que estos hombres sentían por predicar el evangelio, se unía una inmensa fe en el progreso, en la democracia y en las virtudes de la temperancia, el ahorro, el trabajo y, sobre todo, en la educación. Una visión de las sociedad caracterizada por el liberalismo más puro, que descansaba en el individualismo más marcado era el trasfondo de la enseñanza misionera. No es de extrañar su impacto en ciertos sectores que veían en la nueva religión una manera de incorporarse al México moderno en formación. Pero, y. esto conviene aclararlo, todos los esfuerzos realizados y patrocinados por las juntas misioneras tendian a un fin único: la prédica de la necesidad de la experiencia personal de la salvación en Jesucristo, el hijo de Dios. Alrededor de esta idea gira toda la prédica y la acción protestante y metodista. Establecimiento de escuelas, propaganda por medio de la prensa, atención a los desvalidos, todos eran medios para alcanzar ese solo fin. Y se consideraba que la manifestación material, resultado de esos esfuerzos, era el mejoramiento del individuo y, con él, el de la sociedad.

\section{El problema}

Cuando abordamos el estudio de un grupo protestante, encontramos el gigantesco problema que tenemos los mexicanos respecto a las cuestiones religiosas: la falta de información. Si entendemos que las bibliotecas son un reflejo de la conciencia social, podemos comprender esto más claramente. Tras un somero análisis de los materiales pertenecientes al sistema de bibliotecas de la Universidad Nacional Autónoma de México, de la Biblioteca Daniel Cosío Villegas de El Colegio de México y de la Biblioteca Eusebio Dávalos Hurtado del Instituto Nacional de Antropología e Historia, nos convencemos de lo poco que el protestantismo llama la atención de los lectores. Sin temor a exagerar, se puede calcular que el número de títulos relativos a este asunto no llega a cien en el conjunto de las bibliotecas mencionadas. Da la impresión, además, que los libros llegaron a ellas por casualidad y no por decisión consciente de la dirección. Sólo dos títulos están en más de uno de los acervos consultados. Además de poco, el material es demasiado general para ser útil cuando se quiere profundizar en el estudio. Es notorio que los textos que ahí encontramos son los más amplios y los de mayor perspectiva sobre el tema, pero que también son los menos ricos en matices y peculiaridades. Se presenta el caso de que la información manejada en la mayoría de los textos no es más que un refrito de la presentada en otros. También es frecuente el caso de libros "sobre el protestantismo" basados en el estudio de uno o dos de los grupos que lo forman, cuando no se pasan por alto las diferencias entre ellos.

Ante esta realidad no son de extrañar planteamientos de algunos estudiosos de las cuestiones religiosas en México, quienes ven en la introducción de grupos protestantes un intento de penetración extranjera en 
la sociedad mexicana y un medio - uno más- que utiliza la clase dominarite para justificar y mantener su posición.

La intención de este escrito es dar a conocer las fuentes bibliográficas existentes para el conocimiento de uno de los grupos protestantes más importantes en nuestro país: el metodista.

\section{El material bibliografico}

Cuando menos existen tres rubros bajo los cuales se puede clasificar el material referente al metodismo mexicano: el de las memorias, el de las publicaciones oficiales y el de los trabajos de indole académica.

Las memorias. Este es el grupo más amplio e interesante de todos. Fruto de la experiencia de sus autores, los libros en él incluidos nos muestran algo más que el lado "providencial" del desarrollo de la obra metodista en México. Reflejan también el lado humano de la empresa. Los ejemplos más característicos de este "género" son los escritos de John Wesley Butler, ${ }^{2}$ de Delia M. de Patterson ${ }^{3}$ y de Ethel Thomas, ${ }^{4}$ cada uno de ellos perfecto ejemplo de los subgrupos que se pueden formar en este apartado: a) interpretación que el misionero hace del proceso evangelizador; b) memoria del quehacer de los misioneros, escrita ya sea por ellos mismos o por personas que les fueron muy cercanas, quienes vivieron los procesos descritos y c) historia de las organizaciones e instituciones metodistas, trabajos que son una combinación de autobiografia e interpretación de los hechos reseñados. Cabe señalar que no existen, o mejor dicho no se han encontrado, trabajos de la misma naturaleza escritos por mexicanos, a pesar de que entre los laicos y los predicadores prominentes ha habido algunos de gran preparación y capacidad intelectual. Enseguida nos referiremos a los subgrupos enumerados.

a) Interpretación que el misionero hace del proceso evangelizador. Tal vez parezca abusivo presentar un apartado dedicado exclusivamente a tres libros, uno de ellos admitido con algo de buena voluntad. Pero la importancia de los textos y su influjo en el resto de la literatura sobre el metodismo lo justifica. Los libros que forman este apartado son dos de los muchos escritos por miembros de la familia Butler. Mexico in Transition..., escrito por el padre, William, y la History of the Methodist Episcopal Church in Mexico, redactada por el hijo, John Wesley. El otro libro incluido es el de Frank S. Onderdonk, misionero de la Iglesia Metodista Episcopal del Sur (IMES) en México: A Glimpse at Mexico.

Lo que estos tres libros tienen en común es la autoría de misioneros que vivieron mucho tiempo entre los mexicanos - excepto el primeroy que, podriamos asegurar, se identificaron con lo propio de este país. Recordemos la activa participación de John Wesley Butler en el lobby para presionar a Woodrow Wilson para que sacara los marines de Veracruz. Pero la permanencia de los autores entre los mexicanos no es la única virtud reflejada en los escritos. Los autores participaron en la organización del trabajo misionero y conocieron intimamente los entrete-

\footnotetext{
${ }^{2}$ John Wesley Butler, History of the Methodist Episcopal Church in Mexico. 'Personal Reminiscenses, present conditions and Future outlook, Nueva York, The Methodist Book Concern, c 1918, $156 \mathrm{p}$.

'Delia M. de Patterson, Sketch of Rev. W. M. Patterson, pioneer misionary to Mexico. 1878-1888, Frankfort, Roberts Printing Co., c 1924, 50 p.

+ Ethel Thomas, Escuela Industrial. Escuela Sara Alarcón, México, Casa Unida de Publicaciones, $1960,139 \mathrm{p}$.
} 
lones del mismo. Hay que hacer notar también que estos libros, sin excepción, son la base para los escritos que revisaremos después.

Mexico in Transition... ${ }^{5}$ es el libro que menos satisface los requerimientos para entrar en este apartado. Su autor William Butler, fundador y primer Superintendente (1872-1879) del trabajo misionero de la Iglesia Metodista Episcopal (IME) en México, sólo estuvo en nuestro país seis años, en contraste con los más de cuarenta años que su hijo y Onderdonk vivieron en contacto directo con los mexicanos. El libro dedica únicamente uno de los ocho capítulos que lo forman a la historia de la misión. En realidad la finalidad del trabajo es hacer un análisis de las relaciones Iglesia-Estado en la historia de nuestro país. Publicado en 1892, Mexico in Transition... resultó ser un libro altamente polémico que tuvo algunas reseñas críticas en revistas sobre México dedicadas a la comunidad americana. El trabajo intenta demostrar que la influencia del catolicismo romano ha sido perniciosa para la vida política, moral y espiritual del país, encontrando en ello la máxima justificación para la penetración protestante que es considerada un intento de llevar la "luz a un mundo de tinieblas". 6 Si quisiéramos ver el contraste de la mentalidad protestante y la católica, ambas en sus más recalcitrantes extremos, la lectura de este texto de un "antipapista por convicción" y de la respuesta de alguien de sobrenombre "Adam", serían un magnifico ejemplo. El último capítulo es el dedicado a los inicios del metodismo en México. Las finalidades, los métodos y la planeación del mismo salen a relucir en cada párrafo. En este sentido son ejemplares las reseñas de la compra y de la remodelación de las propiedades en México y en Puebla - el claustro del convento de San Francisco y la Iglesia de Santo Domingo respectivamente-, hasta la fecha citadas en todos los libros de historia metodista.

Uno de los textos más ricos e interesantes sobre nuestro tema es la History of the Methodist Episcopal Church in Mexico de John Wesley Butler. Butler, quien llegó a México en 1876 y acabó por convertirse en el misionero más importante de la IME en nuestro país entre 1884 y 1918 , estuvo en íntimo contacto con los niveles más altos de la misión y del país durante los cuarenta y cuatro años que reseña en su libro. Si bien la concepción, planificación y realización del trabajo de la IME quedan claramente presentados en el texto, la valoración del trabajo queda en el aire. El autor nunca deja de señalar los problemas que los predicadores enfrentaron y tampoco deja de mencionar las ayudas que recibieron. Asimismo es importante el número de anécdotas que más que relatos simpáticos son verdaderos ejemplos de los cambios que la nueva prédica introducía en el país. Pero no existe una real y verdadera preocupación por entender el contexto en que la nueva Iglesia se insertaba. No sabemos nada, por lo que Butler nos dice, acerca del universo material y conceptual en que se desenvuelven los conversos al nuevo credo. Mucho menos se habla de política, aunque sí de políticos, de Porfirio Díaz particularmente, aunque el libro fue publicado en 1918. Tal como lo dijera Karl Schmitt, "parecería como si la historia se hubiese detenido en 1867 ". ${ }^{7}$ Con la lectura de este libro nos damos cuenta

5 William Butler, Mexico in Transition from the Power of Political Romanism to Civil. and Religious Liberty, Nueva York, Hunt \& Eaton, 1892, 321 p.

- Es interesante el título de un libro escrito por John Wesley Butler: Mexico coming into light (México viene a la luz).

"Karl Schmitt "American Protestant Missionaries and the Diaz Regime: 1876-1911", 
que la fundación de centros estratégicos para la difusión del nuevo culto, ubicados en las regiones más importantes del pais, fue interés prioritario de los misioneros. Las zonas ocupadas fueron el eje VeracruzMéxico (con centros de culto en Puebla, Tlaxcala, Orizaba, Miraflores y la ciudad de México); las regiones mineras de los estados de Hidalgo y Guanajuato (con Pachuca, Real del Monte y Guanajuato como centros de operaciones); el Bajío y Oaxaca. Quedamos con la impresión que el ocupar esos lugares era importantísimo. Buen ejemplo son los casos de Guanajuato y Querétaro donde el ostracismo y la violencia fueron utilizados en repetidas ocasiones para expulsar a los "herejes". Otras zonas, por el contrario, fueron receptivas a las enseñanzas misioneras, la región Puebla-Tlaxcala y Oaxaca particularmente, por las posibilidades de modernizar la vida del creyente a través del nuevo culto.

A Glimpse at Mexico ${ }^{8}$ el libro de Onderdonk, posee un valor diferente por varios motivos. El autor, que vivió entre los mexicanos más de cuatro décadas, ve las cosas desde una perspectiva distinta. Es la del trabajador y no la del dirigente de la empresa. No encontraremos, por lo tanto, ideas acerca de la planeación de la misión, sino los resultados del trabajo simples y escuetos. Por otro lado, el trabajo mismo de la IMES difirió del desarrollado por la IME en México. Para la primera, la expansión al sur de la frontera resultó ser algo casi natural en el contexto del crecimiento de la iglesia texana; asimismo, el trabajo propiamente de misión, aquél realizado en el centro de nuestro pais, tuvo muchos altibajos y acabó siendo abandonado en 1919 a raíz del llamado Plan de Cincinatti. ${ }^{9}$ Nada de lo cual pasó a la IME. Hay otra circunstancia especialmente importante en este caso: fue publicado en 1930, doce años después que el libro del segundo Butler, cuando la situación política de México había cambiado radicalmente y en los momentos en que se gestaba el nacimiento de la Iglesia Metodista Mexicana (IMM). Cambia, por lo tanto, la perspectiva del texto. No es un libro totalizador escrito desde la cúpula de la misión que busca hacer propaganda a la labor misionera. Más bien es la palabra final que hace un recuento del trabajo realizado durante más de cincuenta años. Cabe decir que este libro adquiere una relevancia particular cuando nos percatamos que es una de las pocas fuentes que tenemos para conocer las actividades de la IMES en México.

b) Memoria del quehacer de los misioneros. En este apartado se incluyen libros que si bien son de gran utilidad por la calidad de la información que nos proporcionan, tienen el inconveniente de haber sido escritos como recordatorio de lo que ciertas personas hicieron por las misiones metodistas en nuestro país. Aquí se deben incluir textos como el de Clementina Butler ${ }^{10}$ o el de Delia M. de Patterson. ${ }^{11}$ Todos estos libros tienen como característica principal el intento de rescatar

Journal of Church and State, p. 253-277 (no ha sido posible conseguir datos sobre editorial y fecha).

${ }^{8}$ Frank S. Onderdonk, A Glimpse at Mexico, Nashville, Board of Missions, Methodist Episcopal Church, South, 1930, $90 \mathrm{p}$.

9 En 1914, los misioneros norteamericanos se reunieron en Cincinatti, Ohio, para hacer una distribución "económica" del territorio mexicano. El acuerdo preveía el trabajo cooperativo de las misiones y repartía el territorio mexicano en "zonas de influencia" para evitar el desperdicio de recursos.

10 Clementina Butler, William Butler, The Founder of Two Missions, Nueva York, Eaton \& Maines, 1902, 315 p.

"Delia M. Patterson, op. cit. 
del olvido o mejor dicho de conservar la memoria de las actividades de los primeros misioneros en nuestro país. Debido a tal interés, los autores no se preocupan mucho por la comprensión del fenómeno metodista en su totalidad, sino en la medida en que sus respectivos biografiados participaron en él.

El ejemplo más claro es el pequeño folleto que Delia M. de Patterson escribió en 1924. El interés de la autora era recordar a su marido, William M. Patterson, virtual fundador de la misión central mexicana de la IMES. La misionera incluye, por lo tanto, datos biográficos relativos a la profunda raigambre metodista de Patterson. Se extiende también en lo relativo al trabajo del misionero en Sudamérica. Cuando se refiere al trabajo de su marido en la República mexicana, la Sra. Patterson contrasta los magros alcances de la misión central antes de la llegada de la pareja al país con los grandes logros del esfuerzo realizado por ellos durante diez años (1878-1888).

En este tenor se desarrollar todos los textos que puedan caber en el rubro que nos ocupa. Aun el libro del obispo Gilbert Haven, Mexico, our next door neighbor, ${ }^{12}$ que tiene la misión de la IME como personaje central, se pierde en el detalle y olvida lo sustantivo del trabajo misionero: ¿a quiénes pudo atraer la prédica?

c) Historia de las organizaciones e instituciones metodistas. Como ya se ha apuntado, este subgrupo es una combinación de los dos anteriores. Por un lado es una historia como cualquiera otra, con su aporte de datos y de interpretación; por el otro, y debido a que los autores son personas que por lo general participaron durante muchos años de su vida en las organizaciones historiadas, son una especie de autobiografía. Muchos téxtos se diferencian de los incluidos en el primer apartado porque aquéllos implican reflexión y evaluación de la labor desempeñada, mientras que en éstos encontramos solamente la crónica de los sucesos. Hay una característica importante en este apartado y es que aquí encontramos por primera vez textos realizados por autores mexicanos, lo cual es reflejo de algo muy sencillo: desde muy temprano en la historia de las misiones, nacionales engresados de las instituciones misioneras se incorporaron al trabajo de su alma mater.

Aun así, el ejemplo sobresaliente de este tipo de material es el libro escrito por Ethel Thomas sobre la Escuela Industrial de Santa Julia, posteriormente Escuela Sara Alarcón. ${ }^{13}$ La señorita Thomas, quien dirigió las actividades de la escuela y del internado adjunto, publicó la muy bien documentada historia de la institución en 1960. El motivo: que no quedara en el olvido la labor educativa de una de las escuelas más importantes del metodismo mexicano. Es natural entonces, y esto se siente en cada línea, el apoyo en los recuerdos, en el archivo y en el diario de la autora. Pero la parte más interesante del texto es la primera, donde la misionera se refiere a la época en que ella no estaba en la escuela, porque Miss Thomas recurre al archivo de la fundadora y primera directora de la escuela, Laura Temple, y cita muchas de sus cartas para apoyar sus aseveraciones. Como resultado, encontramos algunas páginas sensacionales sobre la vida en una escuela para niñas y señoritas durante la tormenta revolucionaria. La obra llega a convertirse en un espejo que refleja la vida cotidiana de la ciudad de México durante

${ }^{12}$ Gilbert Haven, Mexico, our next door neighbor, Nueva York, Abingdoñ Press, 187\%,

13 Ethel Thomas, op. cit. 
la época. Pero no encontramos por ningún lado la relación que existe entre los hechos importantes del momento y el gobierno y la administración de la escuela. Si quisiéramos preguntarnos por la forma en que el problema religioso del veinte afectó la addministración escolar, por ejemplo, no encontrariamos respuesta. Peor aún, si indagáramos por la forma en que la autonomía de la Iglesia Metodista afectó la vida de la escuela, tampoco obtendríamos pista alguna.

Otros escritos que cabrían en esta categoría son los de Levi B. Salmans sobre la Obra Médica en Guanajuato ${ }^{14}$ y el de Nannie Holding sobre las misiones patrocinadas por las mujeres metodistas en el norte del país. ${ }^{15}$ Asimismo habria que incluir aquí los muchos folletos sobre escuelas como el Instituto Normal México, el Instituto Mexicano Madero, y otros de autores mexicanos.

Publicaciones oficiales. Los metodistas se han preocupado, mal que bien, por conservar el recuerdo de su pasado. El mejor ejemplo es que en su Disciplina ${ }^{16}$ texto normativo de la organización de la Iglesia Metodista de México (IMM), se incluye una introducción a la historia denominativa. Desafortunadamente esa introducción no es más que un pequeñísimo resumen de los grandes textos "oficiales" sobre la historia del metodismo mexicano.

Esos que llamo textos "oficiales" son los que se han publicado con cierta regularidad - veinticinco años- para conmemorar los diferentes y respectivos aniversarios de la fundación de su iglesia: bodas de plata, bodas de oro, bodas de diamante y centenario. Existe otro libro sobre el cincuentenario de la autonomía y unificación de los metodismos mexicanos publicado en 1980 . Y hay otro tipo de obras que sin ser "versión oficial" de la historia, son elaborados por las autoridades como parte de las conmemoraciones respectivas.

a) Los libros conmemorativos. Tal vez el libro más conocido sobre nuestro tema sea el de las Bodas de Diamante del metodismo en Méxi$c o .{ }^{17}$ No hay estudio histórico sobre el asunto, escrito después de 1948, que no se refiera a este atractivo y profuso libro. Basado en gran parte en los textos ya descritos de los misioneros norteamericanos, el de las bodas de diamante nos presenta una imagen muy completa del significado del metodismo para nuestro país. La Iglesia en sí, sus organizaciones e instituciones pasan revista en estas páginas. No sólo eso, sino que también encontramos relaciones biográficas de todos los obreros metodistas en México hasta el momento de la publicación. Su inconveniente es obvio. Es un intento de resaltar el valor y la importancia del metodismo mexicano. No hay interés en entender a la Iglesia como un fenómeno inserto en algo más complejo, que es la sociedad. Cuando terminamos esta lectura sabemos qué pasó en la Iglesia, pero nunca sabremos a qué cambios sociales respondió.

b) Las colecciones conmemorativas. El otro tipo de los textos llamados oficiales es aquel que incluye discursos y escritos preparados para las celebraciones. Si bien no se puede decir que estos trabajos aporten

14 Leví B. Salmans, Medico Evangelism in Guanajuato, Guanajuato, ed de autor, 1919, $315 \mathrm{p}$.

15 Nannie Holding, $A$ decade of Mission Life in Mexican Mission Homes, Nashville, Methodist Publishing House, 1895, $111 \mathrm{p}$.

16 Disciplina de la Iglesia Metodista de México, México, s/e, 1984, 301 p.

17 Bodas de Diamante del metodismo en México, México, Imprenta Nueva Educación, 1948, $302 \mathrm{p}$. 
gran cantidad de datos e información sobre los hechos históricos, sí son muy útiles para entender la mentalidad con que los metodistas se han abocado a realizar sus empresas.

El representante más notorio es el de Las Bodas de Oro de la Iglesia Metodista Episcopal en México. ${ }^{18}$ Esta es una -así lo definieron los editores- "colección de las producciones literarias pronunciadas en la magna celebración" del cincuentenario de la IME, 1925. El temario abarcado va desde la exaltación de "lo que el metodismo ha hecho en México en cincuenta años" hasta el recuerdo cariñoso de algunos miembros importantes de la misión, pasando, por supuesto, por la breve historia de cada organización en particular. La principal virtud es que los autores son todos personas que conocen intimamente el asunto del que tratan y que además aman su trabajo profundamente. El problema es el mismo que hemos mencionado en cada caso: sólo llegamos a conocer la imagen que se deseaba dar, nunca nos acercamos a la vida cotidiana del metodismo.

Los trabajos de índole académica. Pasemos al último grupo. Está formado con los trabajos escritos por investigadores o estudiosos pertenecientes a instituciones de educación superior. Aquí encontramos los materiales más controvertibles y ricos en matices y cuestionamientos, resultado de que los autores no son metodistas necesariamente, o de que tienen en mente algo más que el simple y agradecido recordatorio de los hechos. Generalmente existe el propósito de justificar en la experiencia histórica el quehacer religioso del momento presente, independientemente de que buscan obtener su grado académico. Son ejemplos los estudios de Clotilde Falcón de Náñez ${ }^{19}$ y de Jean Pierre Bastian. ${ }^{20}$

La señora Náñez intentó un trabajo del tipo reseñado en las memorias del quehacer misionero. Siendo ella amiga personal del obispo Alejandro Ruiz, y habiendo sido su marido intimo amigo del obispo Eleazar Guerra, la autora intentó una breve pero sustanciosa historia de los obispos metodistas mexicanos. No dejó fuente posible sin estudiar, llegando a entrevistar a algunos familiares de sus biografiados y revisando archivos privados. Esto da una extraña dimensión a sus escritos: apoyo documental a sus aseveraciones, cosa rara en historias sobre el metodismo mexicano en 1970. Su problema: se concentra demasiado en el personaje y olvida el contexto en el que desempeña su actuación.

Jean Pierre Bastian se interesa, por el contrario, en un planteamiento netamente político: la Iglesia no ha permanecido al margen de los sucesos públicos de nuestro país y aun ha tomado actitudes de avanzada, participando activamente en movimientos populares como la Revolución mexicana. La virtud del trabajo de Bastian es que redescubre para los metodistas mexicanos el trabajo de archivo. De ahí se desprende la mayor parte de la información que el autor maneja. Estudios sobre los propagandistas protestantes de la Revolución o sobre el metodismo y la clase obrera son buenas aportaciones en este sentido. El problema de los escritos de Bastian es que su marco epistemológico le impide consi-

I8 Las Bodas de Oro de la Iglesia Metodista Episcopal en México. México, Casa Unida de Publicaciones, 1925, $215 \mathrm{p}$.

19 Clotilde F. de Náñez, Five Bishops of the Methodist Church of Mexico, 1830-1971, trabajo presentado como parte de los requisitos para el curso Christianity in Latin America, Dallas, Southern Methódist University, 1971, 75 p.

${ }^{20}$ Jean Pierre Bastian, Protestantismo y sociedad en Mexico, México, Casa Unida de Publicaciones, 1984, 241 p. 
derar elementos de juicio indispensables para una justa evaluación de los hechos historiados. ¿Cómo entender el desarrollo de una Iglesia marginal sin considerar siquiera las caracteristicas de la experiencia religiosa que predica?

También dentro de este grupo se deben incluir trabajos que a últimas fechas se han preocupado por el estudio del fenómeno protestante y su relación con la historia de México, como el trabajo de Deborah Baldwin. ${ }^{21}$ Aqui habría que colocar, por supuesto, el muy apreciable trabajo de Gonzalo Báez Camargo (Pedro Gringoire) sobre la Biografia de un templo, ${ }^{22}$ que rescata el rico pasado del local ocupado por el "cuartel general" del metodismo "norteño" - perteneciente a la IME norteamericana- en México, el claustro del exconvento de San Francisco.

\section{A manera de conclusión}

Sin pretender ser exhaustiva, esta revisión del material bibliográfico relativo al metodismo en México nos ha servido para una cosa: saber que existe material para conocer la acción protestante en nuestro país. Nos ha permitido también establecer ciertos criterios para evaluar los escritos: cantidad de información que presenta, calidad de la información of recida, relación que se hace de los procesos descritos con el todo social en que se desenvuelve la acción estudiada. Después de una cuidadosa lectura de lo existente, nos percatamos de la necesidad de abrir nuevas fịentes de información, principalmente archivos documentales. De la misma manera es necesario dar un nuevo enfoque a la investigación. Hasta hace poco tiempo, la historia del metodismo corría paralela a la historia nacional sin encontrar puntos de contacto. Los primeros intentos por relacionar uno y otro procesos, presentados en los trabajos de los misioneros norteamericanos del siglo pasado, se perdieron con la relectura y reelaboración de sus testimonios.

21 Deborah Baldwin, Variations Within the Vanguard. Protestants in Mexican Revolution. Ph. D. Dissertation, Chicago, University of Chicago, 1979.

${ }^{22}$ Gonzalo Báez Camargo, Biografía de un templo, México, Casa Unida de Publicaciones, $1976,157 \mathrm{p}$ : 